\title{
Orientaciones y decisiones del Gobierno Santos en materia de relaciones internacionales en torno de las negociaciones de paz para la terminación del conflicto con las FARC-EP. Una mirada en perspectiva de construcción de paz (2010-2014)
}

\section{Guidelines and decisions laid down by Santos government on issues of international relations about peace negotiations to end conflict with FARC-EP. A peace building perspective view (2010-2014)}

\author{
Edgar Alonso Vera Castellanos (i) \\ Universidad de San Gil, UNISANGIL \\ evera@unisangil.edu.co
}

\begin{abstract}
Resumen
El presente artículo tiene como finalidad analizar las orientaciones $y$ decisiones que, en materia de relaciones internacionales, adelantó el exmandatario Santos en el marco de las negociaciones de paz con el movimiento guerrillero de las FARC-EP. Estas se han estudiado con la finalidad de generar conocimiento académico en torno a su incidencia en perspectiva de construcción de paz, durante el periodo 2010-2014. Entre los aspectos más relevantes se destaca que el primer mandatario haya orientado sus gestiones hacia la cooperación internacional buscando su apoyo para la finalización de este conflicto armado, para que las decisiones que se adoptasen en el Proceso de paz respondieran a los lineamientos establecidos en materias como víctimas y justicia. También para la implementación del acuerdo de paz y en la verificación sobre el cumplimiento del mismo, entre otras.
\end{abstract}

Palabras clave: Conflicto armado, procesos de paz, relaciones internacionales, cooperación internacional, construcción de paz.

\begin{abstract}
This article has as purpose to analyze the guidelines and decisions about international relations that expresident Santos laid down in the framework of the peace negotiations with Colombian guerilla FARC-EP. These have been studied with the intention of generating academic knowledge contextualized to its incidence in peace-building perspective, during the period 2010-2014. Among the most relevant issues it is important to highlight that the expresident guided the process towards international cooperation efforts seeking their support for the completion of this armed conflict, so that the decisions taken in the peace process meet the guidelines established in areas such as victims and justice. Also, for the implementation of the peace agreements and the verification of the compliance of the same.
\end{abstract}

Keywords: Armed conflict, peace process, international relations, international cooperation, peace-building.

Articulo: Recibido el 10 de junio de 2019 y aprobado el 29 de enero de 2020 


\section{Cómo citar este artículo:}

Vera-Castellanos, E.A. (2020). Orientaciones y decisiones del Gobierno Santos en materia de relaciones internacionales en torno de las negociaciones de paz para la terminación del conflicto con las FARC-EP. Una mirada en perspectiva de construcción de paz (2010-2014). Reflexión Política 22(44), pp. 55-68. doi: 10.29375/01240781.3636

\section{Introducción}

En materia de paz, como es de público conocimiento, a partir de su posesión el 7 de agosto de 2010 , el expresidente Santos expresó su deseo de buscar una Colombia segura y en paz. De forma explícita manifestó "La puerta del diálogo no está cerrada con llave” (El Espectador, 2010). Desde el inicio de su gobierno contó con el apoyo de varios sectores relevantes, entre ellos la comunidad internacional, que contribuyó con algunos de sus actores en sus acercamientos con la guerrilla de las FARC. Estos contactos exploratorios dieron sus primeros frutos el 26 de agosto del 2012, con la firma de un acuerdo general para la terminación del conflicto y la construcción de una paz estable y duradera. A partir de ese momento se llevaron a cabo negociaciones sobre una agenda de cinco puntos. Estas revisten hoy una importancia mayúscula, pues, como lo consideran algunos especialistas en el tema, las condiciones internas y externas para la terminación definitiva del conflicto estaban dadas (Arbeláez, 2013; Sancho y Bonilla, 2014). De acá que el tema sea de vital importancia para la Ciencia Política. Además, permite visualizar los alcances de este proceso de paz en los ámbitos económicos y sociales; así como en los niveles local, regional y nacional. Ningún colombiano podría sentirse ajeno a las decisiones políticas que se tomaron en La Habana sobre el futuro del país.

En lo relacionado con las orientaciones y decisiones en materia de relaciones internacionales y en torno a las negociaciones de paz, el expresidente Santos fue claro desde un comienzo al señalar que la participación de la comunidad internacional sería clave, no solo por su rol de facilitador, o algún otro, en dichas negociaciones, sino en la construcción de una paz estable y duradera ${ }^{1}$. Se planteó entonces, una nueva política exterior del país, en donde la cooperación y el diálogo serían las guías de las relaciones de Colombia con el mundo; asimismo, se aprovecharían escenarios como el Consejo de Seguridad de la ONU para consolidar el aporte del país al sistema internacional (DNP, 2011). Esta comunidad internacional es primordial en su condición de agente que consolide e impulse la construcción de la paz en este país, especialmente en la implementación del acuerdo final de paz (Borda y Cepeda, 2012). El presente documento plantea el siguiente interrogante: ¿cuál fue el curso de las relaciones internacionales que dio el gobierno Santos en el marco de las negociaciones de paz con las FARC, bajo la mirada en perspectiva de construcción de paz en el periodo del 2010 al 2014 ?

\section{Metodologia}

Basada en el razonamiento inductivo, la metodología acá utilizada es de tipo descriptivo proyectivo orientada a hechos o características pertinentes para determinada investigación. Esto permite realizar cierto tipo de proyecciones sobre el tema estudiado (Niño, 2002). En cuanto a la técnica de recolección de información y análisis empleada, esta se realiza mediante la investigación documental basada, en este caso, en el estudio y análisis, de documentos oficiales del gobierno colombiano, así como de organismos internacionales como la ONU. Se trabajó con dos tipos de información: de un lado, información primaria, representada en documentos y declaraciones oficiales del expresidente de la República y del Alto Comisionado para la Paz; y de otro, información secundaria, consistente en documentos nacionales y extranjeros, tales como libros,

\footnotetext{
${ }^{1} \mathrm{Al}$ respecto, Santos fue claro en afirmar que el respeto, la cooperación y la diplomacia serían las bases de las relaciones internacionales del país. Asimismo, enfatizó que Colombia quiere vivir en paz con todos sus vecinos, haciendo alusión a las difíciles, y a veces hostiles, relaciones que el gobierno del expresidente Álvaro Uribe (2002-2010) sostuvo con Venezuela y Ecuador (Semana, 2010). Para especialistas en temas internacionales, Santos tenía una estrategia de política exterior basada en una agenda internacional mucho más fluida y dinámica que la de su antecesor, Álvaro Uribe Vélez. Igualmente, se sostiene que la visión de Santos se dirigió al redireccionamiento e intensificación de las relaciones internacionales, con el fin de consolidar la inserción del país en el concierto mundial, teniendo como fundamento la liberación del comercio y la integración regional (Vergara, 2012). En consecuencia, Santos inició nuevas relaciones con el mundo, pero también tenía claro que una de su principal bandera, la paz, requería un fuerte compromiso doméstico, así como la activa participación y el compromiso de la comunidad internacional (Medina, 2014).
} 
revistas y artículos (en medios físicos o digitales) escritos por especialistas en el tema.

\section{Marco Teórico}

\subsection{Conflicto Armado}

La guerra es una de las formas más importantes de las relaciones entre las sociedades; fue la primera y con certeza será la última. Esta actividad ha sido objeto de reglamentación por todas las culturas y en todas las épocas, desde Sun Tzu con El arte de la guerra en el siglo V antes de Cristo, se han discutido y planteado una serie de normas para aminorar la violencia de los conflictos armados. En la Antigüedad Clásica y en la Edad Media también se consolidaron leyes de guerra, pero es fundamentalmente a mediados del siglo XIX cuando se estructura el moderno Derecho de los conflictos armados.

El posterior desarrollo del Derecho de los conflictos armados se ha representado en dos ramas: el Derecho de la Haya o Derecho de la guerra y el Derecho de Ginebra o Derecho Internacional Humanitario (DIH). El primero trata sobre la conducción de las hostilidades y combates. En términos generales, comprende las tres convenciones del 29 de julio de 1899 y las trece convenciones del 18 de octubre de 1907. El segundo versa sobre la protección de la población civil no combatiente y las víctimas de los conflictos armados, y se encuentra establecido en las cuatro convenciones del 12 de agosto de 1949 y en dos protocolos adicionales del 10 de junio de 1977 .

El DIH realiza una distinción entre los conflictos armados internacionales, en donde se enfrentan dos o más Estados, y los no internacionales, que se presentan entre fuerzas del gobierno y grupos armados no gubernamentales, o entre estos grupos solamente (CICR, 2008). En cuanto a un conflicto armado no internacional, para el DIH es clave estudiar dos referencias jurídicas en aras de determinar su concepto: el Artículo 3 común a los Convenios de Ginebra de 1949 y el Artículo 1 del Protocolo adicional II de 1977. Particularmente, esta última referencia jurídica establece una definición puntual de este tipo de conflictos, y por tanto se aplica a aquellos

Que se desarrollen en el territorio de una Alta Parte contratante entre sus fuerzas armadas y fuerzas armadas disidentes o grupos armados organizados que, bajo la dirección de un mando responsable, ejerzan sobre una parte de dicho territorio un control tal que les permita realizar operaciones militares sostenidas y concertadas y aplicar el presente Protocolo. (CICR, 1977)

El anterior protocolo establece que en el caso de conflicto armado no internacional que surja en el territorio de una de las Altas Partes contratantes, cada una de las enfrentadas tendrá la obligación de aplicar un mínimo de disposiciones relacionadas con las personas que no participen directamente en las hostilidades y con la asistencia de enfermos y heridos en combate. Expertos sostienen que los elementos que configuran un conflicto armado no internacional son: la territorialidad, la posición de fuerzas, el mando o autoridad responsable, el dominio territorial que permita realizar operaciones militares sostenidas y concertadas, y la posibilidad de aplicar el Protocolo II (Valcárcel, 2007).

En el caso del conflicto armado en Colombia, se ha realizado una importante producción intelectual al respecto. Por ejemplo, se plantea el interrogante de si la situación entre el Estado y las FARC era de conflicto armado o de guerra. Para responder a esto, es clave recordar las definiciones básicas. Tomando como referente el ya citado Protocolo II adicional a los Acuerdos de Ginebra sobre los conflictos armados internos se concluye que los elementos que caracterizan una situación de conflicto armado interno aplican con claridad para el caso colombiano (Zuluaga, 2009). Por otro lado, expertos en esta área abordan el conflicto en mención apoyándose en conceptos de algunos académicos. Por ejemplo, el significado de "conflicto" aportado por Peter Wallensteen (1988) es el de una situación social en donde dos partes luchan de manera simultánea por conseguir el mismo conjunto de recursos escasos y de diversa indole (citado por Torrijos, 2009). Así mismo, se puede hablar de la noción de "desarrollo" de Johan Galtung, entendida como aquel estado que se consigue cuando las carencias de los más necesitados se están satisfaciendo y las estructuras culturales y sociales se están transformando en estructuras y culturas de paz (citado por Torrijos, 2009). El desarrollo significa entonces, crear la capacidad para transformar el conflicto. De forma que la paz no solo es la condición que se consigue cuando los conflictos son manejados en una perspectiva creativa y no violenta, sino que, 
precisamente lo que lleva a la violencia es el fracaso en transformar conflictos.

\subsection{Procesos de Paz}

Un proceso de paz es "un esfuerzo para lograr un acuerdo que ponga fin a la violencia, así como para implementarlo, mediante negociaciones que pueden requerir la mediación de terceros" (Fisas, 2010, p. 11); no es un momento puntual, sino una serie de etapas en las que los actores afectados se esfuerzan conjuntamente para lograr acuerdos que permitirán acabar con la situación de conflicto. Se da paso, mediante el diálogo y el consenso, a acuerdos que ponen término a la violencia y a su vez inician "una nueva etapa de progreso y desarrollo que permita superar igualmente las violencias estructurales que propiciaron el surgimiento del conflicto" (Fisas, 2010, p. 11).

Los procesos de paz exitosos comprenden una fase de negociación inicial y cuyos acuerdos deben ser cumplidos:

[Un proceso de paz] no es más que el punto de partida de unas etapas decisivas en las que se verá si realmente el cese de la violencia es capaz de generar una nueva situación de paz positiva, entendida ésta como prosperidad, armonía, desarrollo humano, crecimiento personal y justicia social, entre otros aspectos. (Fisas, 2010, p. 12)

En cuanto a los diferentes modelos de procesos, se distinguen cinco principales: de reinserción, de intercambio, de reparto del poder, de autogobierno y de medidas de confianza. Asimismo, siempre se siguen unas etapas específicas, pero las negociaciones es la que usualmente más tiempo demanda. Por ello, la primera fase es la exploratoria, o de prenegociación, en la que se mide el grado de convicción de las partes, y en donde necesariamente tendrán que ceder algo. Luego viene la fase generadora de confianza en el propio Proceso, en donde se acuerda el papel que pueden jugar terceras partes, se renuncia a la imposición de proyectos y se reconoce al adversario ofreciéndole la legitimidad necesaria para intermediar. Posteriormente, se llega a la fase de negociación, en donde las partes verifican que los interlocutores sean válidos, es decir, que sean los verdaderos voceros de los actores primarios con la capacidad suficiente de tomar decisiones. Si la fase de negociación prospera, se llega a los acuerdos de implementación, a los acuerdos de las formas de verificación, y a los que regulen la manera de resolver otros desacuerdos que puedan emerger en las últimas etapas.

Habrá que gestionar posibles declaraciones de alto al fuego y/o cese de hostilidades, entendida esta última como el alto al fuego más el cese de actividades sobre la población civil (hostigamiento, amenazas, destrucción de infraestructuras, secuestro, etc.), por lo que está vinculado al cumplimiento de las normas del Derecho Internacional Humanitario (DIH). (Fisas, 2010, p. 26)

Si se representan en una pirámide los tipos de actores que intervienen, es posible observar en la cúspide a los actores y a los facilitadores formales. Estos son los más visibles, los que se encuentran en la foto final del acuerdo de paz. En este espectro figura, además, el Consejo de Seguridad de las Naciones Unidas, pero únicamente en los casos en que en su agenda esté incluido el conflicto. En segundo lugar, dentro de la pirámide se ubican actores como aquellos países que acompañan el proceso, los medios de comunicación, las llamadas diplomacias y entidades de apoyo, y los centros especializados en resolución y mediación de conflictos.

\subsection{Relaciones internacionales}

Las relaciones internacionales se pueden definir como relaciones sociales que impactan en la distribución del poder mundial. Estas se pueden explicarse por los sistemas de equilibrio de poder: sistema bipolar rígido, sistema bipolar flexible, sistema jerárquico, sistema universal y sistema de veto unitario. Así, la sociedad internacional es el complejo de relaciones sociales que distribuyen el poder a nivel mundial (Úbeda-Portugués, 2010). El proceso de mundialización de las relaciones internacionales que caracteriza al siglo $\mathrm{XX}$ tuvo como uno de sus más importantes exponentes a la Sociedad de Naciones, principalmente la Organización de las Naciones Unidas (ONU). El origen de la ONU se asocia a la "Carta del Atlántico" que sirvió de soporte a la Declaración de las Naciones Unidas, ratificada por 26 países en plena segunda guerra mundial, en búsqueda de la creación de un nuevo sistema de seguridad ${ }^{2}$. 
Nada más al finalizar la contienda (junio de 1945), la firma en San Francisco de la Carta de las Naciones Unidas y del Estatuto del Tribunal internacional de Justicia oficializaron esa voluntad, que se concretó en la puesta en funcionamiento de la Organización en octubre de ese año, con sede en New York y un total de 51 estados miembros como fundadores. (Méndez, 2011, p. 71).

La necesidad de contactos, exploraciones, negociaciones y diálogos está avalada por el ejercicio diplomático que realizan todos los países a través de diferentes resoluciones del Consejo de Seguridad de Naciones Unidas, y por la definición de establecimiento de la paz que dicha Organización establece.

Al uso de los medios diplomáticos para persuadir a las partes en conflicto de que cesen sus hostilidades y negocien un arreglo pacífico de sus controversias. Como ocurre con la acción preventiva, las Naciones Unidas solamente pueden actuar si las partes en conflicto así lo deciden. El establecimiento de la paz excluye pues el uso de la fuerza contra una de las partes para poner fin a las hostilidades, actividad denominada en el lenguaje de las Naciones Unidas como imposición de la paz. (Fisas, 2010, p. 113).

Dentro del extenso campo de las relaciones internacionales, la ONU, las diplomacias y sus articulaciones con los conflictos y los procesos de paz, es relevante mencionar dos órganos de suma importancia: La Corte Penal Internacional (CPI) y el Derecho Internacional Humanitario (DIH). La CPI fue constituida a instancias de la ONU el día 17 de julio de 1998, y es un organismo judicial dentro de la estructura de lo que se podría llamarse un "gobierno mundial”. Con su creación ya no se trata solamente de la idea de paz mundial con una ley mundial, sino de la materialización de un anhelo de la comunidad universal. En cuanto al Derecho Internacional Humanitario (DIH), este se origina en el impacto de la degradación de las confrontaciones armadas sobre la sociedad civil, la gestión que durante varias décadas desarrollaron las comunidades, a través de organismos internacionales y no gubernamentales en procura de protección, y en Henry Dunant, que hizo posible esta normatividad. Este cuerpo normativo tiene dos sentidos: por un lado, la forma en que se han de conducir las guerras (métodos bélicos, empleo de proyectiles y armas, etc.), y por otro, la exclusión de la población civil de las matanzas y demás aberraciones que ocasiona un conflicto armado (Ballén, 2010).

Otro componente importante dentro de las relaciones internacionales es la comunidad internacional. Al hablar de ella se distingue entre: los organismos multilaterales (ONU, OEA, UE, etc.), los Estados, las ONG internacionales que manifiestan interés en un proceso de paz, personalidades de relevancia en el país, la jerarquía de la iglesia católica, organizaciones internacionales de los partidos políticos, tanques de pensamiento expertos en asuntos de paz, entes como el Banco Mundial y el Banco Interamericano de Desarrollo, además de los países facilitadores o que tengan que jugar otro papel (Borda y Cepeda, 2012).

\subsection{Cooperación internacional}

Con la creación de la Organización de las Naciones Unidas (ONU), y específicamente en su carta fundacional, firmada el 26 de junio de 1945, se determinan los fundamentos para la resolución pacífica de los conflictos entre países y se materializa la cooperación internacional para que se apliquen los principios de dicha carta. Estos principios se concretan en el reconocimiento de las garantías individuales y de la promoción de los derechos fundamentales, en la Declaración Universal de los Derechos del Humanos de 1948.

La Cooperación Internacional ha sufrido importantes modificaciones en aspectos relevantes como metodología, enfoques y actores. Asimismo, ha pasado de ser "un elemento de ayuda a países pobres para luchar contra el subdesarrollo, a centrarse en la lucha contra la pobreza, lográndose consolidar nuevos enfoques, tales como: participación activa, interés mutuo, partenariado y apropiación por parte de los actores locales" (Álvarez, 2012, p. 286). Igualmente, los demás actores se han diversificado de tal manera que, además de los estados nacionales participan diferentes tipos de entes tales como el de sectores privados, el de organizaciones no gubernamentales, la sociedad civil, los departamentos, municipios y mancomunidades, entre otros. De esta forma, 
la Cooperación Internacional se ha venido constituyendo en una herramienta fundamental de los Estados para conseguir mejoras en las condiciones de vida de las personas.

La Cooperación para el Desarrollo Económico, OCDE, esta se entiende como:

Acción conjunta para lograr el desarrollo económico y social de un país, gracias a la ayuda de organizaciones internacionales, organismos no gubernamentales, países con igual o mayor nivel de desarrollo y de la sociedad civil, mediante la transferencia de tecnologías, conocimiento, experiencias o recursos. (Mejía y Hernández, 2013, p. 14).

\subsection{Construcción de Paz}

La construcción de paz puede definirse como el conjunto de acciones para el corto, mediano y largo plazo que hacen posible a una sociedad prevenir, gestionar y resolver los conflictos por medio de sus capacidades, sin tener que hacer uso de la violencia. Análogamente, es un proceso dinámico en donde las personas dentro de la sociedad, al igual que la sociedad en conjunto, fortalecen su capacidad para crear desarrollo sostenible y la seguridad; todo ello realizable a través de la constante mejora estructural en aspectos políticos, económicos y sociales (IECAH, 2010).

En la agenda para la paz el secretario general de la ONU, Boutros Boutrus-Gali (1992) definió la construcción de paz como las acciones dirigidas a solidificar y fortalecer la paz con el fin de evitar una reincidencia en el conflicto (como se cita en Rettberg, 2013). A partir de este pronunciamiento, la actividad de construcción de paz ha crecido y se ha diversificado ocupando áreas como: el desminado, la desmovilización, el desarme y la reintegración, los procesos de reconciliación y reparación, la justicia transicional y el rediseño de diferentes instituciones.

La construcción de paz trasciende la resolución de conflictos por medio de negociaciones de paz o victorias militares porque no involucra exclusivamente a los actores armados enfrentados, sino que implica también a la sociedad civil nacional e internacional en la forma de organizaciones no gubernamentales, sector privado, iglesia y actores internacionales; por la misma razón, su dimensión temporal es más amplia que la de eventuales negociaciones: se inicia mucho antes que un proceso de paz y sus actividades se proyectan hasta bien avanzado el posconflicto. (Rettberg, 2013, p. 17)

Análogamente, el fin de una confrontación no lleva necesariamente a la paz. La falta de confianza y de consensos políticos pueden persistir, al igual que las causas estructurales del conflicto; de tal manera que el logro de consensos y el abordaje de las múltiples causas del mismo necesitan de la atención persistente de actores nacionales e internacionales, incluyendo la sociedad civil local.

Las diferentes experiencias de procesos y acuerdos de paz, como la de El Salvador o la de Guatemala, indican que los actores internacionales pueden ser, a largo plazo muy efectivos si incentivan y apoyan iniciativas de paz locales, regionales y nacionales. Por tanto, el gran desafío para la comunidad internacional es brindar apoyo a los actores locales y nacionales para que cumplan un rol de liderazgo más amplio en sus propios procesos de construcción de paz.

Existen tres ejes de análisis y acción que son inseparables cuando se habla de paz y reconciliación. El primero es la articulación de las iniciativas de los líderes junto con los representantes de los grupos combatientes, la diplomacia internacional y el alto gobierno. El segundo es la integración de las diferentes dimensiones del conflicto, desde lo personal, lo interpersonal, lo comunitario y lo ideológico, hasta el sistema que envuelve a toda la sociedad. El tercer eje consiste en pensar la construcción de la paz y la reconciliación como un esfuerzo por medio del cual hay que responder estratégicamente a los desafíos a corto plazo, tales como escaladas de violencia; a mediano plazo, como la gobernabilidad y el desarme; y a largo plazo, como la reconciliación (Lederach, 2007).

En los conflictos contemporáneos, la construcción de la paz depende de las realidades subjetivas y empíricas que determinan las necesidades y expectativas de las personas, así como la respuesta a dichas realidades. "Es precisamente en este punto donde el paradigma conceptual y la práctica de la construcción de la paz deben alejarse significativamente de las actividades y el marco tradicionales que constituyen la diplomacia de Estado" (Lederach, 2007, p. 58). La naturaleza y las características del conflicto contemporáneo obligan, entonces, a pensar en unos enfoques y conceptos que miren más allá de la diplomacia de Estado tradicional. 
Construir la paz en los conflictos actuales requiere un compromiso a largo plazo para establecer una infraestructura que abarque todos los niveles de la sociedad, permita potenciar los recursos de la propia sociedad para la reconciliación y rentabilice al máximo la contribución externa. (Lederach, 2007, p. 24)

\section{Orientaciones y decisiones del expresidente Santos en el ámbito internacional en el marco del Proceso de Paz (2010-2014)}

\subsection{Fase exploratoria: diseño y estructura de los diálogos de paz con las FARC}

Los procesos de paz se desarrollan en tres fases o etapas: la de prenegociación o exploratoria, que se realizó en La Habana entre febrero y agosto de 2012; la de negociaciones de paz que se iniciaron en Oslo el 18 de octubre, desarrolladas en La Habana, y que se esperaba que condujeran a la finalización del conflicto y a la implementación del acuerdo final de paz; y la fase exploratoria que abarcó de septiembre de 2010 hasta agosto de 2012. Los acercamientos preliminares se dieron con la asesoría del gobierno noruego, país que otorga especial relevancia a la promoción de la paz como un propósito básico de su política exterior

Está dada la importancia del gobierno cubano y el gobierno venezolano, dada su influencia en el conflicto colombiano. Por esto, desde el comienzo del Proceso, el gobierno del expresidente Santos solicitó la colaboración de ambos para facilitar los acercamientos iniciales con los jefes de las FARC (Martínez, 2014). Paralelo a esto se realizó una fuerte actividad parlamentaria para generar las condiciones legales necesarias que dieran viabilidad a la propuesta de paz y reconciliación: el Marco Jurídico para la Paz, integrado básicamente por cuatro leyes y dos reformas a la Constitución. Los tópicos más importantes de ese marco fueron: la Ley de víctimas y Ley de restitución de tierras, en las que se reconoce la existencia del conflicto armado interno, se otorga a las FARC un estatus político, y se consagran los derechos de las víctimas; la Ley de justicia y paz; y, el Marco para la justicia transicional.

Se determinó que Cuba y Noruega serían los países garantes, mientras que Venezuela $\mathrm{y}$
Chile desempeñarían el rol de acompañantes. La agenda de negociaciones de paz se componía de cinco ejes temáticos: (1) Desarrollo agrario integral, (2) Participación política, (3) Fin del conflicto y (4) Solución al problema de las drogas ilícitas y víctimas (Gobierno Nacional y FARC-EP, 2012).

\subsection{Cronología del desarrollo de la fase de prenegociación (2010-2012) y los paises que intervinieron}

El 27 de agosto de 2012, el entonces presidente Juan Manuel Santos anunció a los colombianos que, seis meses atrás había dado inicio a conversaciones secretas con las FARC. Una serie de importantes acontecimientos, de carácter jurídico y político, se dieron desde el inicio de la fase exploratoria y el comienzo de las negociaciones, anunciadas por las partes el día 18 de octubre de 2012. De acuerdo a lo comunicado por la Corporación Humanas Colombia (2012), entre noviembre de 2010 y enero de 2011 se inician contactos entre el expresidente Santos y los representantes de las FARC. Con la ayuda del presidente de Cuba, Raúl Castro, se realiza en Rio de Oro (Cesar) la primera reunión entre el Gobierno y las FARC. Los representantes ante el Gobierno Nacional fueron Alejandro Eder y un funcionario de Acción Social. Como voceros de las FARC, Andrés Paris y Rodrigo Granda. En febrero, en el Estado de Barinas (Venezuela) y con el apoyo de Hugo Chávez, se llega al acuerdo de que las conversaciones formales entre las partes se llevarsen a cabo en Cuba y Noruega. Para el mismo mes, en Cuba, se acordó que las decisiones que se tomasen entre las partes deberían llevar al fin del conflicto, y que la mesa de diálogos no podría ser eterna y etérea.

Para agosto y septiembre de 2012 el Gobierno y las FARC llegan a un acuerdo sobre la agenda de las negociaciones, y Santos le comunica al país que la fase exploratoria con las FARC ha finalizado con la rúbrica del Acuerdo general para la terminación del conflicto y la construcción de una paz estable y duradera, dando a conocer los cinco puntos de la agenda para los diálogos. Asimismo, el 18 de octubre de 2012, se instalan los diálogos de paz en Oslo (Noruega), y el 13 de noviembre se anuncia que el comienzo de las negociaciones entre las partes no será el jueves 15, sino el lunes 19 de noviembre (Corporación Humanas Colombia, 2012). Aunque se manifestó que Cuba y Noruega 
ejercerían como garantes, mientras que Venezuela y Chile lo harian como acompañantes, en el Acuerdo general se dejó la puerta abierta para la participación de más países: "de acuerdo con las necesidades del proceso, se pondrá de común acuerdo invitar a otros” (p. 2).

Hasta el año 2013 era notoria la ausencia de la ONU en el Proceso. no obstante, el expresidente Santos había expresado que era probable que esa organización intergubernamental pudiese ser llamada en la etapa de verificación de los acuerdos y del postacuerdo, en virtud de su experiencia en esta materia, al igual que en construcción de paz y resolución de conflictos.

\subsection{Cronología de la etapa de negociación (2012-2014) y la comunidad internacional}

Las conversaciones entre el Gobierno y las FARC para llegar al fin del conflicto se iniciaron en noviembre de 2012 en La Habana. Santos apostó a unas negociaciones de paz que tuvieran unos criterios diferentes a los adoptados en anteriores procesos: no se decretó una tregua previa, se reunieron los actores invitados a la mesa de negociación y se creó el Marco Jurídico para la Paz. Para agosto de 2013 la comunidad internacional hizo un importante anuncio: "el coordinador residente de las Naciones Unidas en Colombia, Fabrizio Hochschild, declaró que estas podrían ayudar en la verificación de los acuerdos de paz" (Fisas, 2014, p. 65). Uno de los puntos más difíciles en la negociación con la guerrilla fue el papel que desempeñaron organismos judiciales internacionales; para el segundo semestre de 2013, la Corte Penal Internacional (CPI) dirigió una carta a la Corte Constitucional de Colombia en la que sostenía que, en el marco de las negociaciones de paz con las FARC, el perdón por ciertos crímenes o penas muy bajas, daría potestad a dicha Corte para ejercer su jurisdicción en el país.

El 24 de septiembre de 2013, en preparación de su intervención ante la Asamblea General de las Naciones Unidas, Santos solicitó ante los organismos internacionales el apoyo al Proceso de paz en Colombia. También pidió una reunión con la fiscal de la Corte Penal Internacional, Fatou Bensouda, quien había formulado unas recomendaciones por fuera de sus atribuciones a las Cortes de Colombia (Medina, 2014). Luego, en las visitas que realizó a partir del 3 de noviembre de 2014 a España, Portugal, Francia, Alemania, Inglaterra y Bélgica, fue enfático al manifestar que estaba buscando respaldo a su idea de consolidar un fondo que, por medio de la Unión Europea, ayudase a financiar el postacuerdo. En una de sus visitas a Francia, a comienzos de enero de 2015, el mandatario francés le manifestó que, independientemente de la ayuda que pueda proporcionar Europa, Francia desbloquearía recursos de la Agencia Francesa para el Desarrollo, con el fin de contribuir a programas de sustitución de cultivos, derechos humanos y educación rural (Abdahllah, 2015).

La comunidad internacional y diferentes personalidades se manifestaron a favor del Proceso de paz en Colombia. La tercera semana de febrero de 2015 el país recibió un conjunto de apoyos internacionales en la mesa de conversaciones: el respaldo de dos nobeles de paz, Oscar Arias y Kofi Annan, además de la designación, por parte del gobierno de Estados Unidos, de un enviado especial para los diálogos de paz de La Habana, Bernard Aronson (Semana, 2015).

\section{Perspectivas de la comunidad internacional en la construcción de paz en el marco de las negociaciones Gobierno- FARC}

El rol que desempeñó la comunidad internacional en la implementación de los acuerdos y la construcción de paz fue fundamental. En criterio de expertos, esto es clave en un escenario de postacuerdo, pues identifica en la intervención internacional como garante. Sancho y Bonilla (2014) consideran que la amplia experiencia de algunos actores de la cooperación internacional en materia de justicia transicional, reconciliación, construcción de la memoria histórica y trato a las víctimas es fundamental para la materialización exitosa del acuerdo final de paz. Es de destacar que Colombia contaba con un escenario internacional favorable para el desarrollo de las negociaciones paz, dado el respaldo que los países manifestaron. Por eso fue importante para Colombia sacar provecho de la coyuntura y poner fin al conflicto. En palabras de Sancho y Bonilla, sirve para que el país "utilice los aprendizajes derivados de las experiencias internacionales en materia de construcción de paz y permita que la comunidad internacional desempeñe un papel clave durante la transición" (2014, p. 62). Santos asumió la internacionalización de la paz en estrecho vínculo con la cooperación 
internacional, eje central de la política exterior. Esto se da en virtud que dicha cooperación aporta a la construcción de paz, los recursos, pero también el respaldo necesario a las partes involucradas en el conflicto armado interno, al generar seguridad y confianza.

A raíz de la institucionalización de las ejecutorias dirigidas a la demanda y oferta de cooperación, se destacó uno de los soportes de la política exterior del expresidente Santos: la Agencia Presidencial de Cooperación Internacional de Colombia, APC, (Decreto 4152 de noviembre de 2011). La cooperación internacional es una herramienta clave en la consolidación de la paz de Colombia, complementando el presupuesto nacional para financiar los costos que acarree el postacuerdo y, de manera especial, ofreciendo conocimientos y experiencias para "crear capacidades y competencias en Colombia para construir paz” (Giraldo, 2014). Sin embargo, se debe entender que la construcción de una paz estable y duradera es, ante todo, un compromiso y responsabilidad de todos y cada uno de los colombianos. Por ello, la cooperación internacional se limita a complementar los esfuerzos del Estado, pero de ninguna manera reemplaza sus obligaciones.

Durante el periodo de negociaciones de paz varios académicos, como García (2015) y Giraldo (2014), afirmaron que la comunidad internacional apoyará el postacuerdo, pues este generará buenos dividendos en términos de desarrollo humano y social, en virtud, según el expresidente Santos, de que silenciar los fusiles significará.

Recuperar enormes extensiones del campo colombiano, pues Colombia es considerada por la FAO uno de los ocho países en el mundo que pueden aumentar significativamente su producción de alimentos y, en la medida en que las tierras recuperadas se vuelvan productivas, estaremos contribuyendo a la seguridad alimentaria del planeta. (Giraldo, 2014)

Otros autores se refirieron al apoyo de la comunidad internacional durante el postacuerdo en Colombia. Es el caso de Jean-Marie Guéhenno (2015), director ejecutivo del International Crisis Group, quien, en primera instancia, hizo un llamado a la sociedad civil, a las partes en conflicto y a la comunidad internacional, para que se prepararan para hacer frente al postacuerdo. Según Guéhenno (2015), el papel de los actores internacionales durante este podría ser en forma de apoyo financiero, verificación y apoyo político (citado por Quiroga, 2015). Sin embargo, resaltó el citado director ejecutivo, deben ser las partes en conflicto las que acordarán el papel que irá a tener la intervención internacional en el postacuerdo. Igualmente, sostuvo el especialista, es importante el apoyo regional al Proceso de paz; por tanto, sugirió "crear una comisión con participación internacional que supervise la implementación de los acuerdos de paz en su conjunto, una vez estén suscritos" (citado por Quiroga, 2015, p. 2).

Guéhenno afirmaba que en toda intervención internacional debe haber un balance entre ayuda política y ayuda técnica. La ayuda política, en el caso de Colombia, podría estar dirigida a la reintegración de las FARC, a través de un programa colectivo de reintegración, reconciliación y justicia transicional; aspectos enfocados, básicamente, en la propiedad de la tierra, pues este problema está ligado con el inicio del conflicto armado interno. Por su parte, la ayuda técnica podría estar enfocada en la dejación de armas, el proceso de desminado y en la verificación del cese al fuego (Guiroga, 2015).

Con respecto al que podría jugar la comunidad internacional en la etapa de postacuerdo, es relevante destacar que la situación durante el periodo de gobierno de Santos es diferente a la de anteriores mandatarios. Años atrás, en la presidencia de Andrés Pastrana, los recursos de cooperación se incrementaron de forma considerable. Adicionalmente, la situación de la cooperación internacional era caracterizada por el compromiso de la mayoría de los países donantes de incrementar su Ayuda Oficial al Desarrollo (García, 2015). Durante el gobierno de Santos la situación cambió. Se argumentó que Colombia tenía un crecimiento económico sostenido, los niveles de exportación y de inversión extranjera habían aumentado y el país hacía gestiones para ser miembro de la Organización para la Cooperación y el Desarrollo Económico (OCDE). Al contrario, los países tradicionalmente donantes fueron afectados por la crisis financiera de 2008 y por fuertes políticas de austeridad, que los obligó a reducir sustancialmente los recursos de ayuda externa, concentrándolos en las naciones más necesitadas y con indicadores económicos más modestos que los de Colombia (Semana, 2014). 
García (2015) sostuvo que:

La posible firma de un acuerdo para poner fin al conflicto armado interno, si genera un interés suficiente para que los principales cooperantes aporten recursos, pero probablemente serán limitados, puntuales, algunos canalizados a través de programas ya en curso y otros solo para la etapa inicial de implementación del acuerdo. (p. 7).

Sin embargo, independientemente del monto de los recursos económicos que pudiera aportar la comunidad internacional, Mejía y Hernández (2013) sostuvieron que esta podía tener un papel clave en la etapa de postacuerdo. Ese papel no tiene que ver tanto en términos económicos, sino, más bien en términos de calidad,

gracias a aspectos como la celeridad, la agilidad y la flexibilidad en la movilización de los recursos, la transparencia en la asignación, su presencia en territorios de difícil acceso, las lecciones aprendidas de otros procesos de postacuerdo, el reconocimiento como un actor de paz o un actor neutro y la legitimización del proceso de paz. (García, 2015, p. 7)

La cooperación internacional puede tener un peso significativo en varias dimensiones del postacuerdo; pues los donantes distinguen entre necesidades de corto plazo, en el inmediato conflicto, y las necesidades de mediano y largo plazo. En las primeras, se encuentran los recursos para apoyar las reformas legislativas e institucionales que se requieren para la implementación del acuerdo, el desarme, la desmovilización, las misiones de verificación y la reintegración, entre otros, y acá es necesaria una respuesta con experiencia y conocimiento de procesos comparables. En las necesidades de mediano y largo plazo, los donantes identifican aspectos concernientes a "la implementación de nuevas políticas estructurales que la cooperación internacional puede facilitar y apoyar bajo el liderazgo del gobierno" (García, 2015, p. 7). Además, la cooperación internacional juega un papel relevante en la implementación de los acuerdos a nivel local. Para García (2015) esto obedece a que dicha cooperación podría mitigar, en las zonas de conflicto, las debilidades institucionales a través de procesos de fortalecimiento institucional local. De esta forma, dicha cooperación internacional hace presencia en regiones apartadas del país, pero mientras se tomaron las medidas necesarias para la implementación de los acuerdos, el Estado pudo apalancarse y apoyarse en ese trabajo, así como en experiencias previas de los cooperantes en otros países.

Otro elemento a tener en cuenta, fue el relacionado con la eficacia en la ayuda que pueda ofrecer la comunidad internacional para la implementación del Acuerdo:

En varias reuniones del Foro de Alto Nivel de Eficacia de la Ayuda, Paris (2003), Accra (2008) y Busán (2011), donantes y países receptores acordaron mejorar la eficacia de la ayuda para tener un mejor impacto en el desarrollo. Esto contrarresta el hecho de que los recursos de cooperación internacional son limitados y valida el rol que puede tener a nivel internacional. (García, 2015, p. 7)

Los organismos cooperantes, en sus proyectos y previendo el postacuerdo, podrían realizar una tarea de coordinación y búsqueda de sinergias, de forma que un buen número de dichos organismos trabajó en temas que se negociaron en Cuba, como es el caso de la Atención a víctimas y el Desarrollo rural y de tierras:

Estos dos sectores son los que concentran el mayor número de cooperantes y la mayor parte de estos recursos. Otros sectores de particular relevancia para el postacuerdo reciben menos atención de parte de la cooperación internacional. Ante este panorama un ejercicio de división de trabajo entre los cooperantes parece imponerse. (García, 2015)

También se sostuvo que otro factor destacable, dentro de la cooperación internacional en un escenario de construcción de paz en Colombia, es el hecho de que, para lograr la eficacia y la división del trabajo entre organismos cooperantes, se hacía necesario un liderazgo por parte del Gobierno Nacional, a través de una institucionalidad seria y responsable. Las diferentes experiencias internacionales demuestran, según García (2015) "la importancia de un liderazgo del gobierno en esta etapa frente a los donantes y la alineación de la ayuda a las prioridades definidas para el postacuerdo" (p. 7).

Otros medios se formularon la pregunta acerca de qué clase de cooperación internacional necesitaba Colombia después de la firma de los 
acuerdos con la guerrilla de las FARC. Al respecto, la respuesta se centró, según esos medios, en cuatro clases de cooperación: (1) una cooperación internacional que contribuyese a la construcción de paz con un enfoque territorial; (2) una cooperación internacional que tuviese como punto de partida los derechos de las víctimas a la verdad, la justicia, la reparación y garantía de no repetición; (3) una cooperación internacionalqueincluyese, reconociese y garantizase la participación de las mujeres y sus procesos organizativos en la construcción de la paz; y por último, (4) una cooperación internacional que pudiese hacer visible los impactos del modelo económico de desarrollo (Las Dos Orillas, 2015).

A su vez, para apoyar la paz, además del respaldo al Estado para tareas como la implementación de los acuerdos de paz, los diferentes recursos de la cooperación internacional deberían concentrarse en cinco áreas: (1) el fortalecimiento de la sociedad civil, seguimiento y monitoreo al cumplimiento de los acuerdos; (2) el respaldo al apoyo participativo de políticas públicas para superar los diferentes tipos de desigualdades; (3) la acción humanitaria en el proceso de transición, y (4) la defensa de los derechos humanos, la protección de sus defensores y líderes sociales y de paz ( Las Dos Orillas, 2015).

Otro elemento fundamental que podría aportar la comunidad internacional a la construcción de paz, con base en las gestiones que, a nivel internacional, realizó el expresidente Santos durante la etapa de negociación con la guerrilla de las FARC, fue el relacionado con la legitimidad. Se afirmó, que en casi todos los procesos de paz que se han realizado en los países con conflicto armado interno, la comunidad internacional ha tenido una presencia y unas ejecutorias importantes. En el caso particular de Colombia, sostiene Miguel Arturo Fajardo (2015), que desde el presidente Guillermo León Valencia hasta Juan Manuel Santos, se venían negociando recursos internacionales para la paz, pero también recursos internacionales para la guerra. A manera de ejemplo, argumenta Fajardo, el "Plan Colombia" era un plan para el fortalecimiento de la fuerza pública, en tanto la propuesta del expresidente Santos fue, fundamentalmente, un plan para la consolidación de paz.

En aras del bien supremo de la paz, Santos realizó un recorrido internacional por América Latina, Norteamérica y Europa con el propósito de fortalecer el Proceso, y particularmente su construcción, en el periodo de postacuerdo. Esta situación, según el citado académico, fue de suma importancia en la medida en que la opinión internacional, representada en entidades como la ONU, la OEA, la Comunidad Andina de Naciones y UNASUR, entre otras, tiene un peso político significativo y, por ende, aporta un valioso respaldo al proceso de paz a nivel internacional.

Ese respaldo, continua Fajardo, tuvo un impacto en dos sentidos. Por un lado, hizo sentir en los colombianos la necesidad de vivir en paz, y por otro, hizo que se legitimase altamente el Proceso. Al legitimarse, el mensaje para el Gobierno Nacional y para las FARC era claro: la comunidad internacional está de lado de la negociación política del conflicto armado interno y, por tanto, de la construcción de una paz estable y duradera, puntualiza Fajardo (2015). Vale decir, la construcción de la paz tendría una valiosa legitimidad internacional, en términos de recursos como de confianza.

Así pues, todas las políticas, planes, programas y proyectos que se vayan a implementar y ejecutar para la construcción de paz en el postacuerdo, tendrían el relevante respaldo económico y político de la cooperación internacional. El respaldo en términos económicos, si bien, como se mencionó atrás, podría ser modesto, sería fundamental para la reconciliación entre los colombianos.

\section{Conclusiones}

Desde el inicio de su primer gobierno el expresidente Santos estableció unas pautas claras en relación con el aporte que la comunidad internacional pudiera ofrecer para la consecución de la paz con las FARC-EP, en aspectos como una fórmula política que posibilitara el fin del conflicto armado interno y la construcción de fórmulas jurídicas que hicieran fácil la salida política, ello ajustado a los requerimientos en relación a los derechos humanos.

El exmandatario estableció unos criterios; por ejemplo, que la cooperación sería uno de los soportes de las relaciones de Colombia con el mundo. Asimismo, resaltó que además del compromiso de los colombianos, la decidida participación y compromiso de la comunidad internacional, serían fundamentales para la consecución de la paz. Tanto en la fase exploratoria del Proceso de paz como en su fase de negociación, el expresidente determinó unos parámetros con el fin de que la comunidad 
internacional tuviera activa participación. Por tanto, el respaldo internacional en los diálogos de paz y en el postacuerdo fueron determinantes para la legitimidad del mismo.

Sin embargo, siendo la construcción de la paz una tarea compleja, y teniendo en cuenta el papel relevante que pueda jugar la comunidad internacional, es de vital importancia, dada la heterogénea composición de esta y los intereses que tienen sus diferentes actores, que el Estado colombiano limitase racional y estratégicamente la participación de cada uno de estos actores, de manera que contribuyan específicamente en una área determinada, en función de aportar a las demandas y desafíos que implica la transición de violencias arraigadas a la paz. No hay duda de que, en lo que respecta a las orientaciones y decisiones del expresidente Santos ante la cooperación internacional, estas guardaron total coherencia, tanto en el proceso de paz como en la construcción de la misma. Así pues, el exmandatario direccionó sus gestiones hacia esta cooperación, recabando su apoyo para la finalización de este largo conflicto armado interno, para que las decisiones que se adoptaron en el proceso de paz respondieran a los principios y lineamientos establecidos en materias como víctimas y justicia; igualmente, para la implementación del acuerdo de paz en la etapa de transición que se conoce como postacuerdo, y en la verificación sobre el cumplimiento del mismo.

Hay que tener presente que la cooperación internacional únicamente complementa los esfuerzos del Estado, y de ninguna manera los reemplaza. Por ello es clave no olvidar que por muy buena voluntad y recursos que los actores internacionales puedan aportar a la construcción de paz en el postacuerdo, en el país se requieren otros insumos para alcanzar la paz. Uno de ellos es el nivel de solidez del Estado y sus instituciones, pues de nada sirve una logística internacional para el postacuerdo si los gobiernos, en sus diferentes niveles, no tienen la voluntad o no cuentan con las herramientas para lograr los objetivos de reconstruir el país.

En cuanto a las Cortes Internacionales, estas serían fundamentales en la medida en que inciden notoriamente en la consolidación de la paz en Colombia, pues podrían desconocer las decisiones de la justicia nacional, como, por ejemplo, amnistias. De manera que uno de los campos de acción de la construcción de paz en el postacuerdo, la justicia transicional, es el objeto de críticas y sanciones por parte de la comunidad internacional. Esto llevaría a una deslegitimación del proceso que le restaría no solo credibilidad sino esperanza a las víctimas, razón de ser de esa justicia transicional. La reconciliación, objetivo último de esta justicia, quedaría seriamente amenazada. Así entonces, la comunidad internacional tiene un papel relevante como garante o verificadora para que la justicia transicional sea una herramienta efectiva de construcción de paz.

Finalmente, será necesaria una nueva educación para un nuevo país. Una auténtica revolución para formar en valores ciudadanos, una educación que transforme la manera de pensar de las personas. En esta educación, la comunidad internacional jugaría un papel muy importante en la medida en que su trabajo académico e investigativo en este tipo de asuntos, así como su experiencia, serían uno de los apoyos para entender la educación como "un proceso de acción-reflexión en el que las personas son invitadas a participar activamente en el desarrollo y la aplicación de prácticas y estrategias para la construcción de paz" (Lederach, 2007, p. 162).

\section{Referencias}

Abdahllah, R. (2015). Francia y el después de la guerra. El Espectador. Disponibe en: http://www.elespectador.com/noticias/politica/francia-y-el-despues-de-guerra-articulo-540180.

Álvarez, S. M. (2012). Una introducción a la cooperación internacional al desarrollo. REDUR, 285-309.

Arbeláez, Á. (2013). El contexto del proceso de paz con las FARC. Analecta política, 4(5 Jul-Dic), 219 223. Recuperado el 12 de abril de 2015, de http://revistas.upb.edu.co/index.php/analecta/article/ view/2062/1886

Ballén, R. (2010). Los males de la guerra. Colombia 19882008. Bogotá: Editorial Temis S.A.

Borda, S. y Cepeda, F. (2012). Proceso de paz en Colombia. Participación de actores internacionales. Bogotá: Ecoe Ediciones.

Comité Internacional de la Cruz Roja [CICR].

(1977). Protocolo adicional II a los convenios de conflictos armados sin carácter internacional. Obtenido de Comité Internacional de la Cruz Roja: https://www.icrc.org/spa/resources/documents/ misc/protocolo-ii.htm

(2008). Cuál es la definición de "conflicto armado" según el derecho internacional humanitario? Recuperado el 24 de julio de 2015, de 
Comité Internacional de la Cruz Roja: https:// www.icrc.org/es/doc/assets/files/other/opinion-paperarmed-conflict-es.pdf

Corporación Humanas Colombia. (2012). Los diálogos entre el Gobierno de Santos y las FARC-EP. Sigue a la 1325(3). Recuperado el 26 de abril de 2015, de http://www.humanas.org.co/archivos/boletinsigue3.pdf

Departamento Nacional de Planeación [DNP]. (2011). Plan Nacional de Desarrollo 2010-2014: Prosperidad para todos. Bogotá: Departamento Nacional de Planeación. Obtenido de https://colaboracion.dnp. gov.co/CDT/PND/PND2010-2014\%20Tomo\%20II\%20 CD.pdf

El Espectador. (2010). "La puerta del diálogo no está cerrada con llave": Juan Manuel Santos. Obtenido de https://www.elespectador.com/noticias/politica/ articulo-217858-puerta-del-dialogo-no-esta-cerrada-llave-juan-manuel-santos

Fajardo, M. A. (Comunicación personal 5 de noviembre de 2015). Comunidad Internacional y Construcción de Paz. (E. A. Vera, Entrevistador).

Fisas, V.

(2010). Alto el fuego. Barcelona: Icaria.

(2014). Anuario de procesos de paz 2014. Barcelona: Icaria.

García, J. (2015). Cooperación Internacional y posconflicto en Colombia: más allá de los recursos económicos. Sextante. Recuperado el 30 de abril de 2015, de https://issuu.com/rfaciso/docs/sextante 5

Giraldo, Á. M. (2014). La cooperación internacional en clave de paz. Semana. Recuperado el 28 de octubre de 2015, de https://www.semana.com/nacion/articulo/ la-cooperacion-internacional-en-clave-de-paz/408680-3

Gobierno de la República de Colombia [Gobierno Nacional] y Fuerzas Armadas Revolucionarias de Colombia-Ejército del Pueblo [FARC-EP]. (2012). Acuerdo General para la terminación del conflicto y la construcción de una paz estable y duradera. Recuperado el 13 de enero de 2015, de Alto Comisionado para la Paz: http://www.altocomisionadoparalapaz.gov.co/procesos-y-conversaciones/acuerdo-general/Documentos\%20compartidos/Acuerdo_General para la terminacion del conflicto.pdf

Instituto de Estudios sobre Conflictos y Acción Humanitaria. IECAH (23 de noviembre de 2010). Construcción de la Paz. Obtenido de Instituto de Estudios sobre Conflictos y Acción Humanitaria: https://www.iecah.org/index.php/investigacioncp

Las Dos Orillas. (10 de agosto de 2015). ¿Cómo puede apoyar la cooperación internacional el proceso de paz? Las2Orillas. Recuperado el 30 de octubre de 2015, de http://www.las2orillas.co/co- mo-puede-apoyar-la-cooperacion-internacional-el-proceso-de-pazl

Lederach, J. P. (2007). Construyendo la paz. Reconciliación sostenible en sociedades divididas. Bilbao: Colección Red Gernika.

Martínez, M. Á. (abril de 2014). Proceso de paz en Colombia. Posibles implicaciones para Venezuela. Recuperado el 12 de abril de 2015, de FES Seguridad: http://library.fes.de/pdf-files/bueros/la-seguridad/10707.pdf

Medina, M. (2014). El rompecabezas de la paz. Medellín: La Carreta Editores E.U.

Mejía, H. V. y Hernández, M. A. (2013). Cooperación Internacional para la construcción de paz: potencialidades en la agenda de cooperación colombiana. Bogotá: Universidad Nacional de Colombia. Recuperado el 22 de octubre de 2015

Méndez, R. (2011). El nuevo mapa geopolítico del mundo. Valencia: Tirant Lo Blanch.

Niño, A. E. (2002). Metodología de la investigación II. San Gil: UNISANGIL.

Quiroga, S. (2015). El día después de mañana: Posconflicto en Colombia en perspectiva internacional. ConPaz. Obtenido de https://conpaz.uniandes. edu.co/es/index.php/component/docman/doc_download/4-relatoria-evento-posconflicto-con-icg?Itemid=

Rettberg, A. (2013). La construcción de paz bajo la lupa: una revisión de la actividad y de la literatura académica internacional. Estudios Políticos (42), 13-36.

Sancho, R. y Bonilla, M. E. (2014). Oscar Arias y el proceso de negociación de paz en Colombia. Bucaramanga: Universidad Autónoma de Bucaramanga.

Santos, J. M. (2010). Discurso completo de posesión de Juan Manuel Santos. Revista Semana.

Semana

(2014). Las cuentas del posconflicto no dan. Recuperado el 15 de enero de 2015, de https:// www.semana.com/nacion/articulo/las-cuentas-del-posconflicto-no-dan/408351-3

(2015). El espaldarazo de Kofi Annan al proceso de paz. Recuperado el 8 de marzo de 2015, de http://www.semana.com/nacion/articulo/kofi-annan-vistia-colombia-apoyo-juan-manuel-santos-proceso-de-paz/418909-3

Torrijos, V. (2009). Crisis, paz y conflictos. Bogotá: Universidad del Rosario.

Úbeda-Portugués, J. E. (2010). Lecciones de relaciones internacionales. Madrid: Aebius.

Valcárcel, J. M. (2007). Concepto de conflicto armado interno y seguridad jurídica. Prolegómenos, 10(19), 107-121. Obtenido de https://doi. org/10.18359/prole. 2548

Valencia, A. (1989). Derecho Internacional y conflicto in- 
terno: Colombia y el derecho de los conflictos armados. Colombia Internacional, 6, 3-9. doi:doi: https://doi.org/10.7440/colombiaint6.1989.00

Vergara, R. A. (2012). Análisis de política exterior en Colombia: gobierno de Juan Manuel Santos, ¿continuación de un proceso o cambio de rumbo? Equidad y Desarrollo(17), 149-175. Obtenido de https://www.researchgate.net/publication/284174753 Analisis_de_politica_exterior_en_Colombia_gobierno_de_Juan_Manuel_Santos_continuacion_de_un_proceso_o_cambio_de_rumbo

Zuluaga, J. (2009). Orígenes, Naturaleza y Dinámica del conflicto armado. En F. E. Velásquez, Las otras caras del poder. Territorio, conflicto y gestión pública en municipios colombianos (págs. 45-96). Bogotá: Fundación Foro Nacional por Colombia. Obtenido de http://www. mamacoca.org/docs_de_base/Cifras_cuadro_mamacoca/gtz2010-0038es-gestion-publica-municipio.pdf 